\title{
The effect of educational program on nurse's knowledge and practices about nasogastric tube feeding at neonatal intensive care units
}

\author{
Amira Adel Mohammed*1, Safaa Abdel Fattah Abou Zed Abdel Fattah ${ }^{2}$ \\ ${ }^{1}$ Pediatric Nursing Department, Faculty of Nursing, South Valley University, Qena, Egypt \\ ${ }^{2}$ Pediatric Nursing Department, Faculty of Nursing, Ain Shams University, Cairo, Egypt
}

$\begin{array}{ll}\text { Received: November 22, } 2017 & \text { Accepted: January 24, 2018 Online Published: } \text { March 20, } 2018 \\ \text { DOI: } 10.5430 / \text { jnep.v8n8p21 } & \text { URL: https://doi.org/10.5430/jnep.v8n8p21 }\end{array}$

\begin{abstract}
Objective: This study was to assess the effectiveness of educational program on nurses' knowledge level and their practices about nasogastric tube feeding at neonatal intensive care units.

Methods: Settings: The study was conducted at Banha Specialized Pediatric Hospital and Banha University Hospital, from neonatal intensive care units. Research design: A quasi-experimental design. Sample: A convenient sample method of eighty three (83) as a one study group. Tools: The questionnaire format, the observation checklist and construction of educational program which were developed by the researchers to assessment for the knowledge and practice for nurses' related to nasogastric tube feeding. The educational program was designed serves as a referral instructional for nurses.

Results: Implied that the educational program was having a positive effect, on enhance nurses' knowledge and practice related to nasogastric tube feeding at neonatal intensive care units.

Conclusions: The present study recommended that an administrating a written protocol of nasogastric tube feeding to assure sufficient knowledge, and saved practices are really needed at neonatal intensive care units.
\end{abstract}

Key Words: Neonatal intensive care units, Nurses' knowledge, Nasogastric feeding, Practice

\section{INTRODUCTION}

The neonatal period is the first 28 days of a newborn life. High risk neonate, regardless of birth weight, size, or gestational age, has increased incidence of morbidity and mortality rate, so that neonate required special care, and admitted to Neonate Intensive Care Unit (NICU). Some reflexes as suckling and swallowing with breathing do not begin to develop until around 32 week's gestation. So, the coordination to maintain nutritional requirements by oral feeding without intervention for premature baby usually not occurred. Nutri- tional requirements are frequently met by the administration of nasogastric tube. ${ }^{[1]}$

A nutritional deficiency is a serious problem for high risk neonates. The enteral nutrition (EN) is the best method to promote adequate nutrition via naso- gastric tube pass from the nose to the stomach. May be pass from the nose is called a nasogastric tube (NG). May be gastrostomy if the tube passes via the skin into the stomach, which important prior nutrition intervention support for prematurity newborns and high-risk neonates, as it supplies nutritional requirements

\footnotetext{
*Correspondence: Amira Adel Mohammed; Email: Amira_ad2000@yahoo.com; Address: Pediatric Nursing Department, Faculty of Nursing, South Valley University, Qena, Egypt.
} 
when newborns have difficulties in oral intake. ${ }^{[2]}$ The prior objective of it is to decrease losses from body storage; it is improvement to supply nutrition requirements to enhance newborns growth and development. ${ }^{[3]}$

Nasogastric tube feeding is the prioritize method of administrating nutrient in the high risk neonates. That provides the healthy chance for the intestinal membrane and has improved the immunity for the intestinal membrane rather than parental nutrition. On other hand the parental nutrition when compared with the nasogastric tube feeding out the second is safer and less expensive. ${ }^{[4]}$ Nasogastric tube is the promote feeding method for infants with any problem at gastrointestinal tract, comatose infant, infants breathing is increased than normal or with the assistance of a mechanical ventilator and premature infants. ${ }^{[5]}$

For infants whose needed short period of feeding, the nasogastric feeding (NG) is the most frequently used method of enteral nutrient particular. The NG tube can inserted for many indications as, feeding or administration the medication. The indications of NG tube for low birth weight child or newborn have from respiratory distress syndrome, neonatal sepsis and complications related to congenital anomalies, dehydration, and electrolyte imbalances. ${ }^{[6,7]}$

Nurses' knowledge and skills that applied nasogastric tube have two very important factors in preventing any defects. Adequacy and convenient nursing intervention and chemistry should be observing are needed for an effective nasogastric tube as a therapy. ${ }^{[8]}$

Nursing responsibility in the intervention of NG are: evaluating neonate, recording for the fluid balance, weighting neonates daily, checking of NG instruction, which including, insertion of the tube, administration of feeding, maintenance of the tube, prevention the complications associated with this type of therapy and must be alert by neonates assessment for their responses to tube feeding. ${ }^{[9]}$

Nurses should be aware by appropriate size for the tube, evaluate right position for the tube, different ways for tube securing, enhance right position for neonatal during administration and after, assessing the tube patency, assessing the amount type of food so it must be a liquid, evaluating the intestinal movement, which are necessary part to reduce the complications of NG tube as malposition feeding tubes continue to surface; most are due to inadvertent placement in the respiratory tract and isolated reports of a nasally placed tube entering the brain following head injury continue to occur, as do reports of esophageal and gastric perforation in neonates and enhance maximum neonatal integrity that is for proper management for NGT. ${ }^{[10]}$
Nursing care quality more affected at neonatal outcomes. So, positive progress in neonatal integrity can be carried out by proper nursing practices. ${ }^{[11]}$ Nursing managements is known by the neonatal needs and consider an adequacy of knowledge, practices and previous background. When nurses have a good comprehension about necessary concepts in nursing skills, help in promote nursing practices. ${ }^{[12]}$

\subsection{Significance of the study}

Recent advances studies have demonstrated sick neonates can't obtain adequate nutrition by the normal way, thus nasogastric tube support is required. In some, the GI tract may have health problems for days to weeks (e.g., necrotizing entero-colitis, GI system anomalies), so the infant receives all feeding by nasogastric tube. Moreover, previous studies had reinforcement the nurses' knowledge and practice about the NG feeding among adult patients at neonates intensive care units. Fewer researches focused the nurses' knowledge and practices related to nasogastric tube feeding in neonatal care units.

\subsection{Aim of the study}

Assess the effectiveness of educational program on the knowledge level and practices for nurses about nasogastric tube feeding at neonatal intensive care units.

\subsection{Research questions}

- Nurses' knowledge level regarding nasogastric tube feeding at neonatal intensive care units will increase.

- Daily nurses' practice regarding nasogastric tube feeding at neonatal intensive care units will improve.

- Nurses who attend the educational program will have a higher mean post-test scores related to knowledge and practices of nasogastric tube feeding at neonatal intensive care units compared to mean pre-test scores.

\section{Methodology}

\subsection{Design and sample}

A quasi-experimental design was used in the current study where the nurses' sample served as their own control (Single group study). A convenient sample was utilized in this study eighty three (83) of available nurses working at the study settings. Practitioner nurses were with various educational categories representing and all should be participated voluntarily in the study.

\subsection{Settings}

The study was conducted at the NICU from the Banha Specialized Pediatric Hospital and Banha University Hospital. 


\subsection{Tools of data collection}

Three tools were used for data collection:

Tool (I): Questionnaire sheets, it was designed by researchers to clarifying the practitioner nurses' knowledge about nasogastric tube feeding at neonatal intensive care units designed in English and it includes:

Part (1): Socio-demographic data of nurses which included open-ended questions regarding age, educational level, attending previous training, marital status nurses' and experience by years.

Part (2): Assess the performance for nurses' related nasogastric tube feeding to assess knowledge of nurses before, during and after nasogastric tube feeding administration. It consists of 15 multiple choices and True/False questions.

Scoring system Regarding nurses' knowledge assessment questionnaire, the incorrect one was scored zero and the right answer was scored 1. They covered knowledge regarding the definition of NGT, indications of NGT, contraindications NGT, and complications of NGT. A total score for the questionnaire was ranging from $(0-15)$ score where adequate at $60 \%$.

- Adequate knowledge: 9-15 scores (60\%).

- Inadequate knowledge: 0 to 8 scores $(40 \%)$.

Tool (II): Follow up observation checklist designed by the researchers to observe nurses' practice before, during and after nasogastric tube feeding administration. It included 30 items.

Scoring system: Follow up observation checklist was scored by Likert scales (not done $=0$, incomplete done $=1$ and done $=2$ degrees). A total score for the checklist was ranging from (0-60) degrees where a cut-scores at $60 \%$.

- Sufficient skills ranged from 36-60 scores (60\%).

- Insufficient skills (<36) scores $(40 \%)$.

Tool (III): Construction of educational program. The evaluation was conducted and the program was then designed. The researchers used different teaching methods during program implementation, for example, lectures, and watching videos and posters for gastric feeding. In addition, an educational booklet has been designed by researchers in Arabic language with regard to gastric feeding. It was considered an educational referral to nurses in neonatal intensive care units. The educational booklet was revised for the validity and clarity of three professional in pediatric nursing. In the light of their comments, the researchers made the necessary adjustments in the final form of the educational manual.

\subsection{Validity and reliability of the study tools}

The study tools were reviewed by five Pediatric Nursing professional, who reviewed the instruments for clarity, relevance, understanding, comprehensiveness, applicability and easiness. In the light of their revision, modifications in the face validity were carried out.

\subsection{Ethical considerations}

Before the conduction of the pilot study as well as the study was approved the directors of Banha Specialized Pediatric Hospital and Banha University Hospital. Consent informed was taken from each nurse will included in the study sample and that participation is voluntary. At the initial interview was taking a sufficient explanation about the study. All information provided will be kept confidential. The participants were informed that there are no known risks or hazards known to be associated with the participation or non-participations in this study.

\subsection{Pilot study}

A pilot-tested for feasibility, clarity and time requires being applies. Done on $10 \%$ from the sample size were 13 nurses. Nurses were included in the pilot study were excluded at the finally from the study sample.

\subsection{Procedure of data collection}

After obtaining an official permission from the directors of Banha Specialized Pediatric Hospital and Banha University Hospital, data were collected over three month's period in 2017. Participants were recruited from the previous mentioned settings. Each nurse was interviewed individually after taken their consent to contribute in the study and was given sufficiency identification about the study aim by the researcher. The first, the researchers beginning to collect the socio-demographic data, after that was assessed nurses knowledge's related to nasogastric feeding tube a pre-test distributed for the studied sample. At this time, researchers were good observed the nurses during their work and complete the follow up observation checklist. The educational program was proceed over 15 demonstrated session was taken around 1 hour at various morning shifts by moderate of 3 sessions/week for one month. The educational program was included group discussion and videos related to the skills regarding nasogastric tube feeding. Immediately after ended the program, using the same tools of pre-test, for the post-test that done to evaluate the program effectiveness. After that, the conclusion and recommendations of the study were distribute to the recruited sample, all nursing staff at the NICU from the Banha Specialized Pediatric Hospital and Banha University Hospitals, with copies of the booklets regarding educational program. 


\subsection{Statistical design}

The data were organized, presented and analyzed by SPSS version 21. Data were evidenced by frequency and percentage, Comparison between experimental and control groups and had done by using Chi-square test $\left(\chi^{2}\right)$ and $t$-test for qualitative data. Significance difference was at $p$-value $<.05$.

\section{RESULTS}

Table 1 shows socio-demographic characteristics of the studied nurses. It was founded the nurses ages were $60.2 \%$ of the nurses were between 30 and 35 years and $14.5 \%$ were over 36 years. Fifty nine percent of the studied sample had between first and fifth years' experience as a practitioner nurse. Nearly thirty nine of the participants had attended previous training courses for one time. Approximately $92.0 \%$ were holding a bachelor degree.

Table 1. Studied sample distribution regarding to sociodemographic data

\begin{tabular}{|c|c|c|}
\hline Items & No & $\%$ \\
\hline \multicolumn{3}{|l|}{ Age } \\
\hline 20-30 years & 21 & 25.3 \\
\hline 30-35 years & 50 & 60.2 \\
\hline More than 36 years & 12 & 14.5 \\
\hline \multicolumn{3}{|l|}{ Marital status } \\
\hline Married & 57 & 68.7 \\
\hline Widowed & 5 & 6.0 \\
\hline Divorced or single & 21 & 25.3 \\
\hline \multicolumn{3}{|l|}{ Educational level } \\
\hline Bachelor & 76 & 91.6 \\
\hline Master & 4 & 4.8 \\
\hline Diploma & 3 & 3.6 \\
\hline \multicolumn{3}{|l|}{ Experience as nurse } \\
\hline Less than 1 year & 4 & 4.8 \\
\hline $1-5$ years & 49 & 59.0 \\
\hline 6-10 years & 16 & 19.3 \\
\hline More than 10 years & 14 & 16.9 \\
\hline \multicolumn{3}{|l|}{ Experience NICU } \\
\hline Less than 1 year & 4 & 4.8 \\
\hline 1-5 years & 53 & 63.9 \\
\hline $6-10$ years & 15 & 18.0 \\
\hline More than 10 years & 11 & 13.3 \\
\hline \multicolumn{3}{|c|}{ Attending previous training } \\
\hline Never & 3 & 3.6 \\
\hline For one time & 32 & 38.6 \\
\hline For two times & 24 & 28.9 \\
\hline More than 2 times & 24 & 28.9 \\
\hline
\end{tabular}

From Table 2 it was clear, researchers were an observed improvement regarding the knowledge of the recruited sample post the educational program. At the same time the results of this study showed that the participants knowledge mean scores before, during and after nasogastric feeding administration were $0.67 \pm 0.843,2.10 \pm 1.226$ and $2.64 \pm 1.384$ degree pre- program. On other hand, post-program mean scores regarding nurses knowledge before, during and after nasogastric feeding administration were $2.658 \pm 0.524,5.0706 \pm$ 1.19312 and $6.294 \pm 1.737$ respectively before, during and after nasogastric tube feeding administration. That reflects the educational program improvement the nurse's knowledge regarding to nasogastric feeding at neonatal intensive care units.

Table 3 illustrated that the studied nurses' practice mean scores before, during and after nasogastric feeding administration were $13.02 \pm 6.378,11.29 \pm 7.281$ and $3.51 \pm$ 2.711 degree pre-program. On other hand, post-program mean scores regarding nurses practice before, during and after nasogastric feeding administration were, $17.223 \pm 2.804$, $13.823 \pm 1.774$ and $6.294 \pm 1.737$. That reflects the educational program competence at the nurse's practice regarding to nasogastric feeding at neonatal intensive care units.

It is evident from Table 4, a highly statistical difference, was presented to compare the level of knowledge scores and practice scores of the studied sample in pre and post program.

Researcher hoping this study's results reached to minimizing gap between research studies and clinical practice and this practical evidence to establish policy on the interventions in neonatal units to decreasing neonatal mortality.

\section{Discussion}

There is a growing importance on good care for neonatal intensive care units. However, studies of nasogastric feeding tube feeding have focused more often on adult intensive care units, and less focused has been given to neonatal intensive care units.

As the present study detected, more than half of the nurses their ages ranged between $30-35$ years. This might be related to their old graduation, this study is similar to Mula ${ }^{[13]}$ found the majority of nurses' ages in his study were ranged between 35 and 45 years. While Shahin \& Mohamed ${ }^{[14]}$ were found that three-quarters of the nurses in their study less than 25 years old. Also Taha \& Mohammed ${ }^{[15]}$ found that half of nurses aged between 26-35 years old.

As the current study implied that fifth nine of studied nurses had experiences were between 1 to 5 years, this study are similar with Metwaly ${ }^{[16]}$ who found that more than one-third of nurses had 1-4 years' experience. Moreover, Mohammed et al. ${ }^{[17]}$ found that more than half of nurses in neonatal intensive care units settings are having more than 10 years of working experiences. 
Table 2. Comparison the mean scores nurses' knowledge's regarding nasogastric tube feeding

\begin{tabular}{lllll}
\hline \multirow{2}{*}{ Items } & $\begin{array}{l}\text { Pre-program } \\
\mathbf{( N = 8 3 )}\end{array}$ & $\begin{array}{l}\text { Post-program } \\
(\mathbf{N}=\mathbf{8 3})\end{array}$ & \multirow{2}{*}{-Test } & $\boldsymbol{p}$ value \\
\cline { 2 - 3 } & Mean $\pm \mathbf{S D}$ & $\mathbf{M e a n} \pm \mathbf{S D}$ & ${ }^{*}-18.247$ & .00 \\
\hline Before administration of feeding & $0.67 \pm 0.843$ & $2.658 \pm 0.524$ & $*-15.817$ & .00 \\
During administration of feeding & $2.10 \pm 1.226$ & $5.0706 \pm 1.19312$ & $*-14.989$ & .00 \\
After administration of feeding & $2.64 \pm 1.384$ & $6.294 \pm 1.737$ & $*$ \\
\hline
\end{tabular}

* Significance difference at nurses’ knowledge’s pre and post-program

Table 3. Comparison the mean scores nurses' practices regarding nasogastric tube feeding

\begin{tabular}{|c|c|c|c|c|}
\hline \multirow[t]{2}{*}{ Items } & $\begin{array}{l}\text { Pre-program } \\
(\mathrm{N}=\mathrm{83})\end{array}$ & $\begin{array}{l}\text { Post-program } \\
(\mathrm{N}=\mathrm{83})\end{array}$ & \multirow[t]{2}{*}{$t$-Test } & \multirow[t]{2}{*}{$p$-value } \\
\hline & Mean \pm SD & Mean \pm SD & & \\
\hline Before administration of feeding & $13.02 \pm 6.378$ & $17.223 \pm 2.804$ & $*-5.496$ & .00 \\
\hline During administration of feeding & $11.29 \pm 7.281$ & $13.823 \pm 1.774$ & $*-3.080$ & .00 \\
\hline After administration of feeding & $3.51 \pm 2.711$ & $6.294 \pm 1.737$ & $*-7.877$ & .00 \\
\hline
\end{tabular}

* Significance difference at nurses’ practices pre and post-program

Table 4. Comparison regarding the nurses' knowledge level and practices

\begin{tabular}{|c|c|c|c|c|}
\hline \multirow[t]{2}{*}{ Items } & \multicolumn{2}{|c|}{$\begin{array}{l}\text { Pre-program } \\
(\mathrm{N}=83)\end{array}$} & \multicolumn{2}{|c|}{$\begin{array}{l}\text { Post-program } \\
(\mathrm{N}=83)\end{array}$} \\
\hline & $r$ & $p$ & $r$ & $p$ \\
\hline $\begin{array}{l}\text { Nurses' knowledge } \\
\text { Nurses' practice }\end{array}$ & 0.078 & .241 & 0.384 & $* .000$ \\
\hline
\end{tabular}

The present study results showed that the bachelor degree was the most of studied participants. This result was disagreeing for the results of Ghoddoos et al. ${ }^{[18]}$ who have found that BSN degree was the majority of participants.

Nearly one third of studied sample revealed had training course at NICUs. This training program helps practitioner nurse to keep up to date on the most recent developments in nursing and to be able to manage the needs of nursing practice. These findings agree with Metwaly ${ }^{[16]}$ who recorded, more than half of participants having training courses. While these results disagree with Shehab ${ }^{[19]}$ who registered that, the majority of nurses did not receive training programs regarding nasogastric tube feeding.

From the present study for the questionnaire sheet which was assessed nurses knowledge, the pre-test analysis clarifying the knowledge about definition and indications of nasogastric tube feeding taken the first highest percentage, complications taken the second highest; care of nasogastric tube feeding taken the third highest, the sources of knowledge for nasogastric tube feeding taken the fourth and contraindications of nasogastric tube feeding, taken the fifth highest before, during and after administering feeding pre educational pro- gram. The study reflected that the nurses' knowledge about nasogastric tube feeding at the neonatal intensive care units was inadequate knowledge. Compared with post educational program before, during and after nasogastric tube feeding administration was adequate knowledge. These findings were agreed with study carried out in the Unite State American; the purpose was to implement an educational program regarding nasogastric tube feeding on 50 critical care nurses from 3 various critical care units to evaluate its effect, the results implied significant increment in mean score of knowledge post program implementation Bourgault et al. ${ }^{[20]} \& \mathrm{Al}$ kalaldeh. $^{[21]}$

From the talking with nurses at the implementation of the educational program, studied sample clarifying that the major the reasons related to inadequate knowledge regarding nasogastric tube feeding were the minimized the hospital instruments and guideline written protocols related to nasogastric tube feeding management at neonates' intensive care units. Shortage knowledge was founded regarding using of internet resources act as a main factor at lack nasogastric tube feeding knowledge.

Related to the nurses' practices at the pre implementation of the program mean scores; before, during and after nasogastric tube feeding administration, the nursing practice pre-program were insufficient, that implied hazards skills form the nurses. Participants form neonatal intensive care units at Banha Specialized Pediatric Hospital and Banha University Hospitals there is no suitable documented guideline or another ways which can obtain specific information's to improve their knowledge and practices regarding nasogastric 
tube feeding administration. On other hand, the practice from nurses' post-program regarding nasogastric tube feeding at neonatal intensive care units from the study settings before, during and after feeding administration were sufficient. The improvement of nurses' practice as a result of implementing an educational or training program was well supported by Kenny, ${ }^{[1]}$ pointed out that there was improvement in all practices of nasogastric feeding tubes administration from feeding and medications' administration after implementing an educational program.

From the discussion of the participants' practices mean score related to nasogastric tube feeding, the present study reflect that the educational program had good effectiveness in the promoting the nursing practice related to nasogastric tube feeding.

In similar study established by Metwaly, ${ }^{[16]}$ in 2 pediatric units at El-Zagazig hospital, Egypt, that was to assess the effectiveness of educational program on knowledge and practices level for nurses' related to congenital hypothyroidism. It was detected, the nurses' knowledge and practice was promoted at post-test.

Regarding the comparison related the knowledge and the practices among the studied sample, the present study re-

\section{REFERENCES}

[1] Kenny D. Care of Patient with nasogastric tube feeding: evidencebased practice protocol. Nursing Research. 2010; 59(1): 22-31. PMid:20010275 https://doi.org/10.1097/NNR.0b013e3181 c3bfe9

[2] Mota M. Evaluation of intensives-nurses' knowledge concerning education administration via nasogastric and enteral tubes. Rev Latino Am Enfermagem. 2010; 18(4): 888-94. https://doi.org/10.1 590/S0104-11692010000500008

[3] Wilson J, Singh B, Dutta S, et al. Guidelines for Nutrients feeding. 2015.

[4] Greenberg D, Bowden R. Pediatric nursing procedures. 1st ed. Lippincott Williams and Wilkins London; 2013.

[5] Schmidt N. Evidence-Based practice for nurses: appraisal and application research. Canada: Jones \& Bartlett Learning International; 2012.

[6] Ahamed N. Assessment of Knowledge and Practice of Staff Nurses regard Ryle's tube feeding in a selected hospital of Kolkata, west Bengal. SM Medical Journal. 2014; 1(2): 294-302.

[7] Persenius M. Assessment of Patient's nutritional status: perception of registered nurses and their chief nurses. Journal of Clinical Nursing 2014; 17: 2125-2136. PMid:18510576 https ://doi .org/10.111 $1 / j .1365-2702.2007 .02202 . x$

[8] Susan S, Arnp P. Essentials of maternity, Newborn and women's health nursing. Lippincott William's and Wilkins, Philadelphia, London. 2012. flect highly statistically among knowledge and practices for the studied sample at pre-implementation and postimplementation for the educational program. This results were expected, the knowledge regarding nasogastric tube is important at the nursing skills.

\section{Conclusion}

Practitioner nurses' level of knowledge and skills regarding nasogastric tube feeding in neonatal intensive care units were inadequate with some serious skills. That due to deficiency of policies, educational instruments and protocols regarding nasogastric tube feeding. The educational program had good effectiveness in promoting where marked nurses' more knowledgeable and promoting skills regarding nasogastric tube feeding at the two previous mentioned settings (Banha Specialized Pediatric and Banha University).

\section{Recommendations}

The study is recommended to administrate a guideline protocol of nasogastric tube feeding, periodically assessment for nurses' knowledge level and practice and establish this study on different hospitals in Banha and Egypt.

\section{CONFLICTS OF INTEREST DisClOSURE}

The authors declare that there is no conflict of interest.
[9] Stacy K, Urden L, Lough M, et al. Thelon's critical care nursing diagnosis and management. 5th ed. St. Louis, MO: Mosby; 2006.

[10] Zhu L. Therapeutic concerns for oral medications are administrating nasogastric-ally. Journal of Clinical Pharmacy and Therapeutics. 2013; 11: 11-15.

[11] Singer P. ESPEN Guidelines on parental nutrition: neonatal intensive care. Clinical Nutrition. 2011; 28: 387-400. PMid:19505748 https://doi.org/10.1016/j.clnu. 2009.04.024

[12] Peterson S, Behara A, Chen Y. Nutrition support in the critically ill. A physician survey. Journal of Parenteral and Enteral Nutrition. 2011; 32(2): 123-129.

[13] Mula C. Assess nurses' knowledge and practice about pediatrics enteral nutrition in special care units of a referral hospital in Malawi. Australian and New Zealand Marketing Academy Conference, Perth WA. 2011.

[14] Shahin M, Mohamed W. Nurses' knowledge and practices regarding nasogastric feeding at the neonatal intensive care units of Al-Manial University Hospital in Egypt: impact of a Designed Instructional Program. Journal of American Science. 2012; 8(9): 397-404.

[15] Taha A, Mohammed E. Critical care nurses' knowledge and practice regarding administration of total parental nutrition at critical units in Egypt. Journal of Biology, Agriculture and Healthcare. 2014; 4(13): $10-22$.

[16] Metwaly E. Nurses' practices regarding nasogastric tube feeding in neonatal intensive care units, Unpublished Master Thesis. Faculty of Nursing Zagazig, University. 2013. 
[17] Mohammed W, Abdullah M, Islam M. Knowledge and practices nurses bout administration of medications by nasogastric tube among critically ill children. Journal of Education and Practices. 2014; 5(2): 137-49.

[18] Ghoddoos I, Ameri Z, Vafaee A, et al. The effect of a total parental nutrition training program on knowledge and practice of nurses in NICU. Global Journal of Health Science. 2016; 8(9): 135.

[19] Shehab M. Assess the nurse's practices in providing care to pediatrics undergoing nasogastric tube. Unpublished Master Thesis, Faculty of
Nursing, Suez Canal University. 2009.

[20] Bourgault A, Ipe L, Weaver J, et al. Development of evidence-based guidelines and critical care nurses' knowledge of enteral feeding. Critical Care Nurse. 2015; 27(4): 17-29.

[21] Al kalaldeh M. Jordanian nurses' knowledge and responsibility for enteral nutrition in the neonatal intensive care units. Nursing in Critical Care. 2015; 20(5): 229-41. PMid:24279734 https: //doi.org/10.1111/nicc.12065 